\title{
Unannounced Evacuation Experiment in a High-Rise Hotel Building with Evacuation Elevators: A Study of Evacuation Behaviour Using Eye-Tracking
}

Axel Mossberg (D*, Division of Fire Safety Engineering, Lund University, Box 118, 22100 Lund, Sweden and Brandskyddslaget, Långholmsgatan 27, Box 9196, 10273 Stockholm, Sweden

Daniel Nilsson, Department of Civil and Natural Resources Engineering, University of Canterbury, Christchurch, New Zealand

Kristin Andrée, Brandskyddslaget, Långholmsgatan 27, Box 9196, 10273

Stockholm, Sweden

Received: 31 January 2020/Accepted: 17 September 2020

\begin{abstract}
Past studies suggest that people are often reluctant to use occupant evacuation elevators in case of fire. However, existing research is scarce and current knowledge is based on questionnaire studies and laboratory experiments. An unannounced evacuation experiment was therefore performed on the 16th floor of a 35-floor highrise hotel building. Sixty-seven participants took part and eye-tracking glasses were used to collect data on exit choice and eye fixations. Three different scenarios were studied, including two different hotel room locations on the floor and a variation of guidance system for one of these locations, i.e., flashing green lights next to the evacuation sign at the elevators. Results suggest that people typically choose the elevator for evacuation, even if their hotel room was located closer to the evacuation stair. Flashing green lights next to an evacuation sign made people look more at this sign. However, in spite of looking more at the sign, the flashing light was not shown to significantly improve compliance with the sign. Also, the results suggest that a detector activated self-closing fire door without vision panels to the elevator lobby made it more difficult to find the evacuation elevators in an emergency.
\end{abstract}

Keywords: Evacuation elevators, OEE, Human behaviour, Exit choice, Eye tracking, High-rise building, Evacuation, Fire

\footnotetext{
* Correspondence should be addressed to: Axel Mossberg, E-mail: axel.mossberg@brand.lth.se
} 


\section{Introduction}

Society is facing new challenges in the built environment as urban populations grow. The growth of urban populations has recently been declared as one of the Societal Grand Challenges of fire safety science by the International Association of Fire Safety Science (IAFSS) [1]. IAFSS acknowledges that both the increase in urban population and the demographics of that population have changed dramatically in recent years and will keep changing in the future, which will affect the fire safety needs of society.

The number of high-rise buildings around the world are undoubtedly increasing. The seven tallest buildings in the world were all built in the last decade [2] and there are more than 25 buildings with a planned building heights over $400 \mathrm{~m}$ currently under construction [3]. At the same time, people over 65 years is the fastest growing population group [4]. These developments demand a review of evacuation procedures for high-rise buildings [1, 5].

Although several studies have shown increased evacuation efficiency for occupant evacuation elevators (OEEs) compared to stairs [6-10], there is still a reluctance to incorporate OEEs in evacuation designs. This is likely connected to the difficulties in predicting human behaviour in an evacuation with such elevators [11]. Currently, guidance on how to address human decision making in these evacuation scenarios is lacking. The prediction of evacuation behaviour in a building with OEEs is complicated by (1) the recommendations in "regular" buildings that elevators should not be used in case of fire, and (2) the Theory of Affiliation postulating that people tend to exit a building using familiar routes [12], which is often the elevator.

Previous studies show a relatively low acceptance of the elevator as an escape route, but also indicate that the willingness to use elevators for evacuation increases higher up in the building [13-15]. These different studies were all based on questionnaires and hypothetical fire scenarios, but they show large differences in the correlations derived between the willingness to use an OEE and the building floor. More recent studies carried out in Virtual Reality (VR) indicate an increased willingness to use evacuation elevators than predicted in the questionnaire studies, and also that this willingness can be further increased when green flashing lights are used to re-enforce the OEE evacuation signage [16]. These results are consistent with previous research on the effects of green flashing lights at emergency exits in other situations, which has suggested that flashing lights next to emergency exit signs are effective at influencing route choice by making people notice the signs and in addition, the colour green has a positive cognitive interpretation as it is associated with safety [17, 18]. It should be noted that other factors have been suggested to affect the willingness to use an OEE, e.g., movement impairments [19] and culture [13]. Also, the effects of body mass index and age on the willingness to use an OEE have been investigated [13].

The use of eye-tracking technology to better understand and investigate human behaviour has increased in recent years [20]. This technology allows researchers (and others) to explore where people look and use this data to investigate behaviours, e.g., the time needed for people to acquire certain visual information [21]. 
Research on eye movement and attention has shown that the eye movement have a central role in how attention shifts [22] and that the attention in turn is a key component in decision-making [23]. Thus, eye tracking can provide valuable insight into human visual perception and has been applied in several different research fields, e.g., the effects of training on inspection of aircrafts [24], how the eye movements when driving are affected by talking on a cell phone [25], or to study user behaviour connected to Internet searches [26]. In fire research, eyetracking technology has been identified as a useful tool to investigate the behaviour during an evacuation [27] and some evacuation studies using eye-tracking has been performed, e.g., investigation of the location of evacuation signage in buildings $[28,29]$. However, more thorough studies applying eye-tracking technology in evacuation research are still scarce.

The differences in results of past studies on elevator evacuation emphasises the need for field studies. Similarly, eye-tracking studies for unannounced evacuations are rare. For this reason, a study was initiated in a building where elevator evacuation was a part of the evacuation design. The objectives of the study are to:

1. explore evacuation behaviour in a building with OEEs,

2. investigate the influence of green flashing lights at emergency exit signs on exit choice,

3. use eye-tracking data to explore the decision-making process in an evacuation,

It is important to note that exit choice connected to elevator evacuation is likely affected by the elevator waiting times, i.e., if waiting times are too long people may reconsider their choice of exit. Thus, the willingness to use OEEs can be considered as a combination of the initial exit choice and accepted waiting time. In this study, only the initial exit choice was studied.

\section{Method}

Unannounced evacuation experiments with partially informed participants were performed in a high-rise hotel building, i.e., the experiments involved initial deception about the true purpose of the research. The participants took part individually and the experiment was not repeated, i.e., each participant did the experiment once. Data was collected using eye-tracking glasses and observations were made by researchers in adjacent hotel rooms. The experiment setting, eye-tracking equipment, studied scenarios, participants, and the procedure are described below.

\subsection{Experiment Setting}

The experiment was performed in a high-rise building in Sweden. The building is 35 floors with mixed occupancy, i.e., hotel and office use. The briefing before and after the experiment was given at the ground floor, but the evacuation part of the experiment was performed on the 16th floor. This is one of the hotel floors as the hotel occupies all floors between the ground floor and the 22nd floor, and the office part of the building occupies the 23rd to the 33rd floor. The top two floors 
in the building houses a sky bar connected to the hotel. The reason that the 16th floor was chosen was to enable comparisons to the VR-study mentioned in the introduction [16].

At the experiment floor, evacuation is possible via an evacuation staircase or via six OEEs. The staircase is reachable through a fire protected compartment, i.e. two fire doors need to be passed from the corridor to reach the stairs. One of the elevators is a fire-fighter elevator and is possible to enter/exit both towards the hotel lobby and directly to the hotel corridor. A floor plan can be seen in Fig. 1. Both the staircase and the elevator lobby discharge directly to the outside on the ground floor.

The elevator lobby is designed as a separate fire compartment and the double door separating the lobby from the corridor is a self-closing fire door. However, this door was held open by a magnet before the alarm went off, i.e., the door was open when the participants arrived at the floor but closed when the alarm was activated. The difference in the appearance of the corridor with the door open/closed can be seen in Fig. 2. In this figure, there is an evacuation sign in the corridor with an elevator evacuation sign (closest to the camera) and a regular sign pointing towards the staircase at the end of the corridor (further away). Also note the fluorescent sign on the lobby door that is visible once the door is closed.

The building was also equipped with a voice evacuation alarm with a message in both Swedish and English containing the same information. The English message was:

Attention please, attention please. There is a fire in the building. Evacuate immediately to nearest emergency exit. The lifts can be used as an emergency exit in the building.

In addition to the evacuation signage and the evacuation alarm, the inside of the hotel room doors was equipped with an evacuation plan and a safety brochure. An information sign was placed in the elevator lobby. The evacuation plan showed the floor plan with the OEEs and evacuation staircase highlighted in green and it gave a short explanation of the evacuation strategy, e.g., that the elevators could be used for evacuation. The same information was given in the safety brochure. The information sign in the elevator lobby stated that the space was a safe area and that one should wait for the elevators. This sign did not light up until the alarm was activated.

\subsection{Eye-Tracking Equipment}

Eye-tracking glasses allows researchers to explore what people look at. In this case, the eye-tracking equipment was used to identify what the participants were looking at to gather information before and during their evacuation. To obtain this data, several different techniques can be used. Currently, the most common method is called pupil centre corneal reflection (PCCR) [30]. This is the method used by Tobii in their Tobii Pro Glasses 2 [31], which was the equipment used in the experiments. 


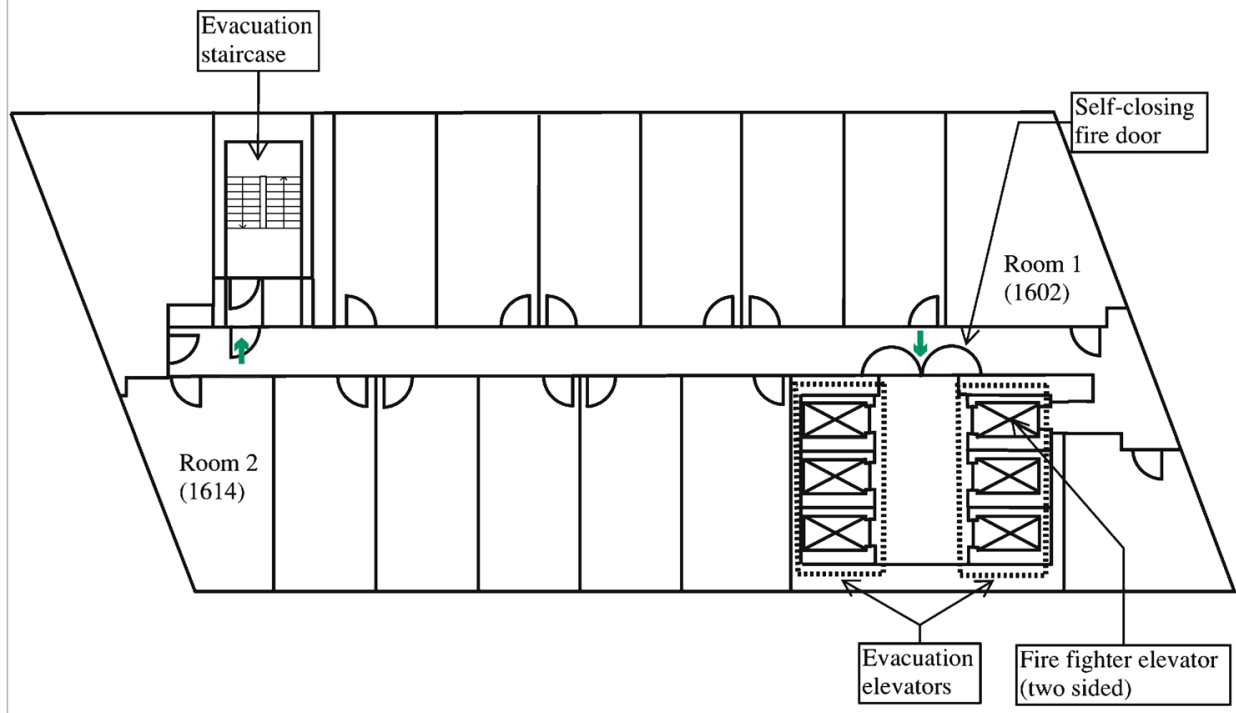

Figure 1. Floor plan of the hotel floor. The two marked rooms (Room
1 and 2) were the ones used in the experiment. The green arrows
indicate the location of the evacuation signs.

Tobii Pro Glasses 2 is a pair of glasses connected to a recording unit. The glasses are equipped with several cameras, both pointed towards the eyes and in the direction of the gaze. This makes it possible to record the eye position over time in a dynamic setting. Also, output can be analysed using the software Tobii Pro Lab [32]. The program allows extraction of detailed data as well as visualization of the results.

The Tobii equipment and software makes it possible to study the fixations of the gaze. A fixation can be defined as the situation when the eye movement stabilise, which is normally assumed to correspond with the desire to maintain the gaze at an object or area [33]. For the classification of fixation data, a velocity threshold identification algorithm (I-VT) was applied, i.e., the I-VT Attention filter was used in Tobii Pro Lab. This filter is recommended when the subject, or the environment, is in constant movement during the experiment [34], which is expected for most of an evacuation experiment. However, this will lead to a $10-15 \%$ overestimation of the fixations, instead of an underestimation if the I-VT Fixation filter would be used. The reason for this is that the velocity threshold, which is the key parameter for fixation classification, is higher in the attention filter $\left(100^{\circ} / \mathrm{s}\right.$ vs. $\left.30^{\circ} / \mathrm{s}\right)$ which will allow some saccades, i.e., the rapid repositioning of the eyes, to be classified as fixations. However, with the I-VT Fixation filter, the fixations would be underestimated as many fixations then would be classified as saccades. Also, short fixations, i.e., fixa- 

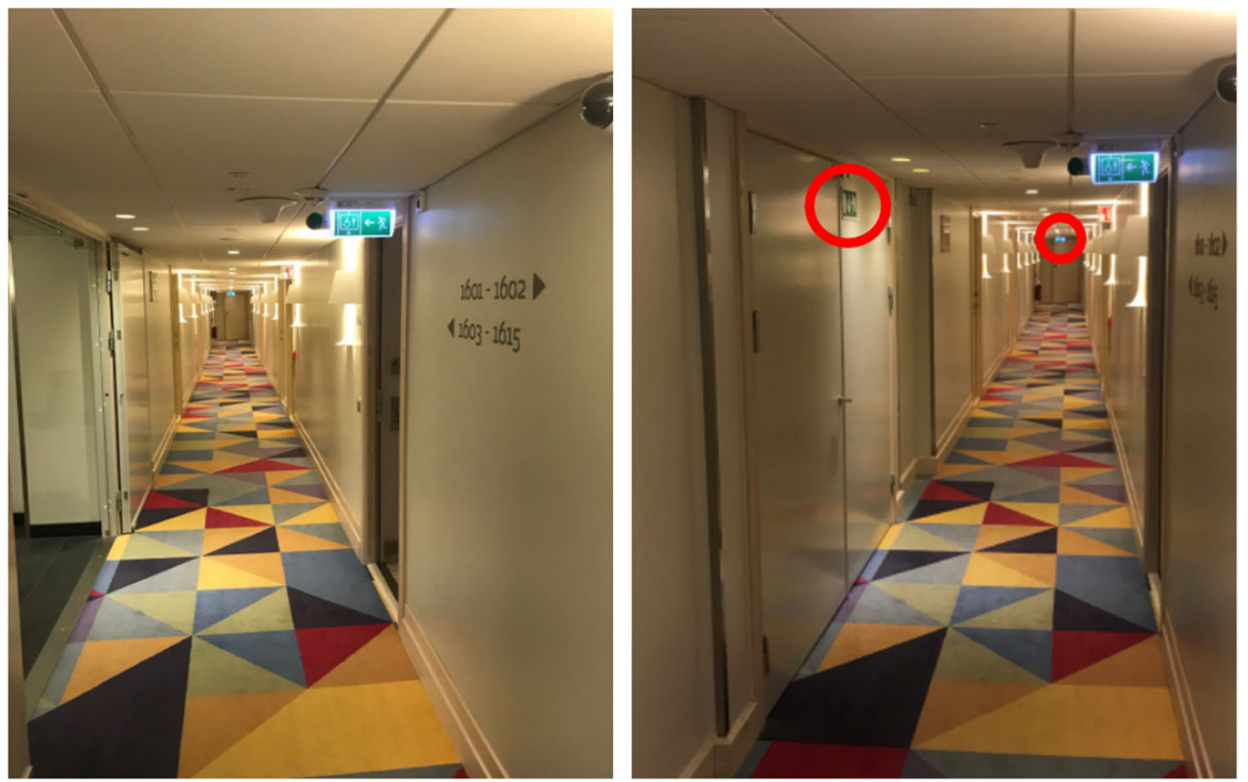

Figure 2. The hotel corridor with the double doors to the elevator lobby open (left) and closed (right). In the right image, the fluorescent evacuation sign on the elevator lobby door and the evacuation sign to the staircase at the end of the corridor has been circled.

tion lengths shorter than $60 \mathrm{~ms}$ were discarded from the data as these fixation length have shown to be too short for the brain to register the information [35].

After the data on fixations have been collected, these fixations were studied by the researchers in a manual post-processing procedure. The fixations of the participants were then displayed as circles layered over a first-person video of the experiment. The researcher could then identify the fixations that were on objects of interest, e.g., evacuation signage etc. The data for these fixations, e.g., fixation times, were then collected in an output file to be further analysed by the researchers.

\subsection{Studied Scenarios}

Three different scenarios were studied in the experiment. These were:

- Scenario 1 -the participant was in room 1 (see Fig. 1) at the start of the alarm and no extra evacuation measures than those described above were used,

- Scenario $1-F L$ - the participant was in room 1 at the start of the alarm, and a flashing light was attached to the evacuation sign pointing towards the elevator lobby (Fig. 3). 


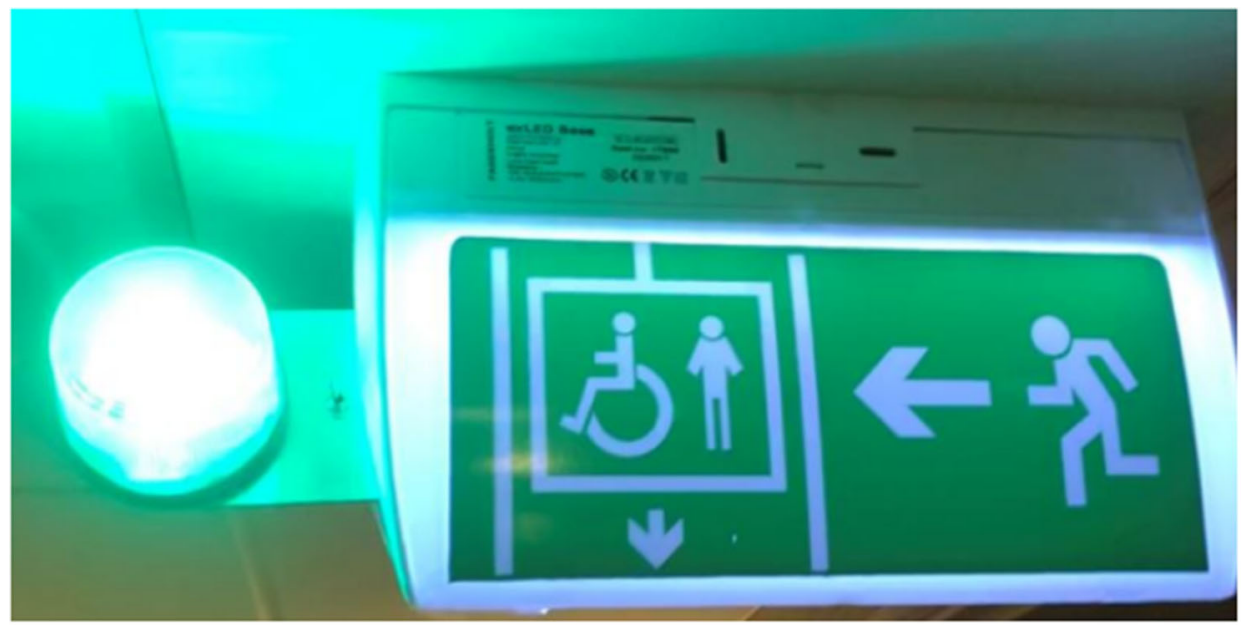

\section{Figure 3. Flashing light attached to the evacuation sign pointing towards the elevator lobby.}

- Scenario 2-the participant was in room 2 at the start of the alarm and no flashing lights were attached to the evacuation signage.

Thus, the scenario name is related to the starting room of the participant, with the addition of the FL for the scenario with added flashing light. The light in Scenario $1-F L$ flashed with a frequency of $1 \mathrm{~Hz}$ which is a frequency based on past studies [18].

The different scenarios studied were chosen mainly to see if (1) the exit choice was affected by flashing lights and (2) if the starting position affected the exit choice. Thus, scenario 1 can be regarded as a base-line scenario and scenario 1-FL and scenario 2 as comparative scenarios.

\subsection{Participants}

Sixty-seven persons took part in the experiment, namely 37 women $(55 \%)$ and 30 men $(45 \%)$. The participants were recruited through a Swedish website for participation in research experiments and they were told they would participate in a hotel design study. The age of the participants ranged from 20 to 71 years old with an average age of 33.2 years $(\mathrm{SD}=11.6$ years). All participants were Swedish, except five persons (from Germany, China, Croatia, Poland and the United States). All participants were given two movie tickets (approximate value of 250 SEK) as reimbursement for their participation. In general, this reimbursement was given to the participants after they had finished the experiment and completed a questionnaire. However, the participants were informed that they would receive the reimbursement even if they decided to terminate the experiment at any time. Only one of the participants answered that they had trouble walking in stairs (this was due to knee arthritis) and none of the participants were frequent visitors of 
the building in which the experiments were performed. The participant characteristics for each scenario is given in Table 1 below.

The number of participants, i.e., the sample size, was mainly determined by the time provided by the hotel for the experiments. Thus, this sample was not previously determined by an a priori sample size calculation.

\subsection{Procedure}

As mentioned above, the participants were recruited through a website for experiment participation. The recruitment information mentioned participation in a design study focusing on how people perceive hotel rooms.

When the participants arrived at the hotel, a researcher met them in the hotel lobby, and they were taken to a conference room. The researcher pointed out that this was a study focusing on hotel room design and the eye-tracking glasses were put on the participant. To increase the likelihood of the participants keeping the glasses on, they were told that the equipment was very expensive, and the glasses were also secured with an extra strap around the head. For participants with the need of corrective lenses, a range of such was available and able to mount in the eye tracking glasses. This option was used by a few participants. No participant used their own glasses under the eye tracking glasses due to the risk of this disturbing the equipment.

Once the participant had been informed about the study, although the true purpose was not revealed, they were asked to sign an informed consent form before participating in the study. The eye-tracking equipment was then calibrated, the participants were given a hotel room key, instructed to go to the hotel room and act as if they had just checked in at the hotel. They were also told that another researcher would come and fetch them in the hotel room in 10-15 min, and the experiment would then be terminated. In the instructions, they were also given a number to call if they wanted to terminate the experiment for any reason. It should be noted that all participants choose to travel to the 16th floor by elevator and thus, all participants entered the floor by elevator through the elevator lobby in Fig. 1.

A few minutes after the participant reached the hotel room, the alarm was activated and thus the evacuation part of the experiment was initiated. The experiment was terminated when the participant had chosen an escape route, i.e., one of

\section{Table 1}

\section{Participant Characteristics for Each Scenario}

\begin{tabular}{lllccr}
\hline & \multicolumn{4}{c}{ Participant characteristics } & \\
\cline { 2 - 5 } Scenario & Women, n (\%) & Men, n (\%) & Total, n & Mean age & Age SD \\
\hline 1 & $12(55)$ & $10(45)$ & 22 & 32.8 & 11.1 \\
$1-\mathrm{FL}$ & $11(50)$ & $11(50)$ & 22 & 31.1 & 10.6 \\
2 & $14(61)$ & $9(39)$ & 23 & 35.6 & 13.3 \\
Total & $37(55)$ & $30(45)$ & 67 & 33.2 & 11.6 \\
\hline
\end{tabular}


with Evacuation Elevators

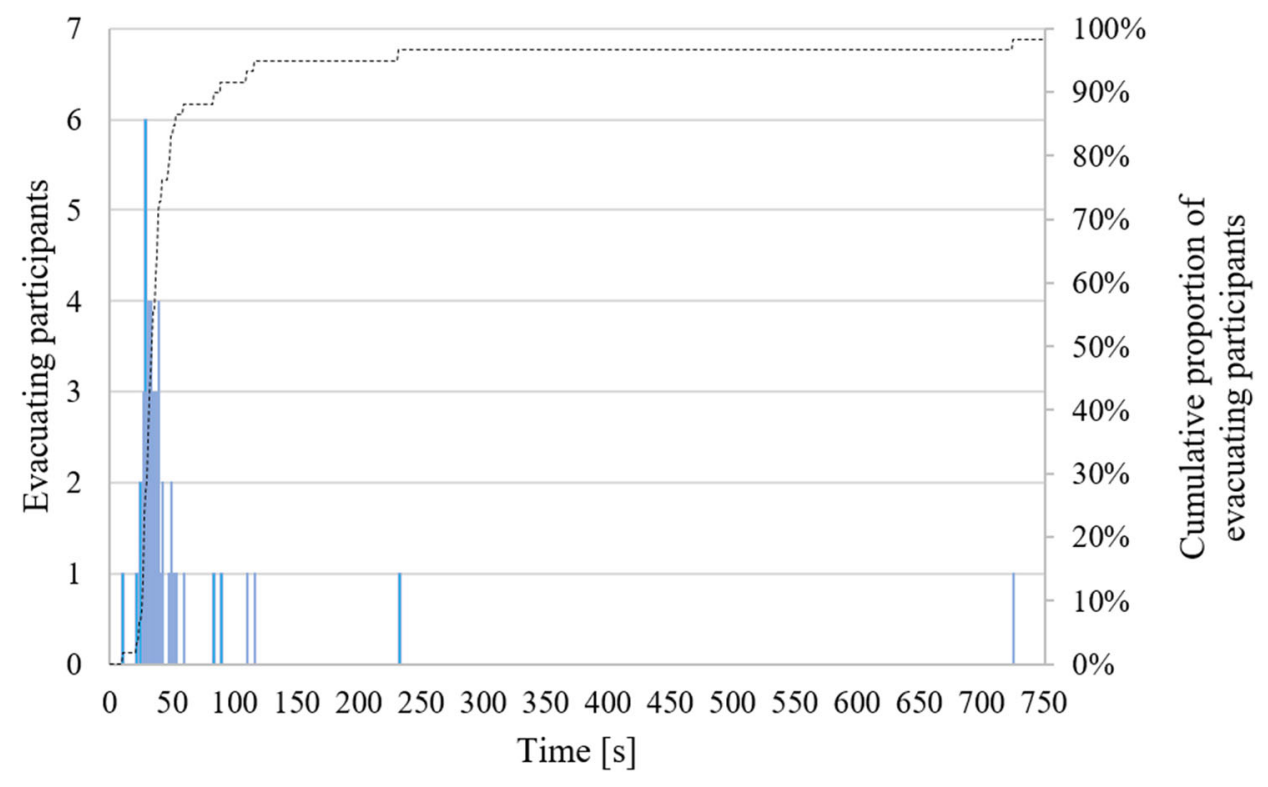

\section{Figure 4. Time until the participants left the hotel room after the evacuation alarm was activated. The left Y-axis show number of participants evacuating each second and the right $Y$-axis show the cumulative proportion of evacuating participants at each time.}

the OEEs or the evacuation staircase. For the participants choosing the staircase, a researcher was waiting in the stairs and for the participants choosing the elevators, a researcher was waiting in an adjacent hotel room, monitoring the participant using a tablet connected to the eye-tracking glasses and through the peephole in the door. When a participant pressed the elevator call button, the researcher in the hotel room revealed him- or herself and terminated the experiment. The participant was informed about the true purpose directly after the experiment and accompanied the researcher to a conference room at ground level. The researchers debriefed the participant and made sure that she/he was feeling okay and then asked her/him to fill out a questionnaire. The questionnaire consisted of background questions about the participant, e.g., gender and age, and questions about their evacuation experience, e.g., if they perceived any risks with the exit choice they made. The data from these questionnaires was later used in the analysis of the experiment. Finally, the participant was asked to sign the informed consent a second time to ensure that they approved of the use of the data from the experiment after knowing the true purpose of the study.

It should be noted that the experiment did not include any investigation of waiting times at OEEs, as a researcher terminated the experiment as soon as the participant had pressed the elevator call button. The reason for this was primarily due to the fact that the rest of the building was in normal use and, thus, it would 


\section{Table 2}

\section{Description of the Primary Exit Choice of the Participants in Each} Scenario

\begin{tabular}{lllccr}
\hline & \multicolumn{5}{c}{ Exit choice } \\
\cline { 2 - 5 } & $\begin{array}{l}\text { Staircase, } \mathrm{n} \\
\text { Scenario }\end{array}$ & $\begin{array}{r}\text { Elevator from corridor, } \\
\mathrm{n}(\%)\end{array}$ & $\begin{array}{c}\text { Elevator from lobby, } \\
\mathrm{n}(\%)\end{array}$ & $\begin{array}{c}\text { Elevator in total, } \\
\mathrm{n}(\%)\end{array}$ & Total \\
\hline 1 & $0(0)$ & $4(18.2)$ & $18(81.8)$ & $22(100)$ & 22 \\
$1-\mathrm{FL}$ & $1(4.5)$ & $2(9.1)$ & $19(86.4)$ & $21(95.5)$ & 22 \\
2 & $2(9.1)$ & $5(22.7)$ & $15(68.2)$ & $20(90.9)$ & 22 \\
\hline
\end{tabular}

have been possible for evacuees to bump into visitors who were not part of the experiment in the elevator.

The participants were alone on the hotel floor (except for the researchers monitoring the experiment) and did not interact with anyone else during their evacuation. The reason for this is further elaborated in the discussion.

\section{Results}

In this section, the results of the experiments are described. IBM SPSS Statistics Software [36] was used for statistical testing. The significance level was set to 0.05 for all tests. Eye-tracking data was only collected for 58 of the 67 participants due to technical difficulties (failing batteries, etc.). Also, one participant did not evacuate the hotel room within $900 \mathrm{~s}$ from alarm activation, when the researchers decided to terminate the experiment. Thus, exit choice was recorded for 66 participants, while data involving eye-tracking was recorded for 58 participants.

\subsection{Pre-evacuation Behaviour}

Before the participant left the hotel room after the alarm was activated, there was no substantial difference in setup between scenarios and thus, no comparisons between scenarios are made. However, the fixations and behaviour of the partici-

\section{Table 3}

Description of the Participants Perception of Escape Routes

\begin{tabular}{llllr}
\hline & \multicolumn{3}{c}{ Noticed more than one escape route? } \\
\cline { 2 - 5 } Scenario & Yes, n (\%) & No, n (\%) & No answer, n (\%) & Total \\
\hline 1 & $4(18.2)$ & $18(81.8)$ & $0(0)$ & 22 \\
$1-$ FL & $3(13.6)$ & $17(77.3)$ & $2(9.1)$ & 22 \\
2 & $8(36.4)$ & $14(63.6)$ & $0(0)$ & 22 \\
\hline
\end{tabular}



with Evacuation Elevators

pants were still recorded and studied. Results show that 3.4\% $(n=2)$ of the participants fixated their gaze on an evacuation sign during normal conditions, i.e., on their way to the hotel room. In addition, $5.2 \%$ of the participants $(n=3)$ read a safety brochure that was available in the hotel room and $8.6 \%$ of the participants $(n=5)$ studied the evacuation plan on the inside of the hotel room door before the alarm was activated.

Once the alarm was activated, the participants left the hotel room within 10 $725 \mathrm{~s}$ with an average time of $54 \mathrm{~s}$ and a standard deviation (SD) of $94 \mathrm{~s}$. The time to leave the room can be seen in Fig. 4. As can be seen in the figure, there are a couple of outliers, i.e., participants taking a very long time to leave the room compared to other participants. Also, one participant did not evacuate within $15 \mathrm{~min}(900 \mathrm{~s})$, at which time the researchers decided to terminate the experiment.

Before evacuating from the hotel room, $43.1 \%$ of the participants $(n=25)$ fixated their gaze at the evacuation plan on the inside of the hotel room door. Also, $84.5 \%$ of the participants $(n=49)$ went to the alarm speaker in the hotel room and fixated their gaze, one or several times, on it as one of their first actions once the alarm had activated. A red information sign with white text stating "Evacuation alarm" was located under the speaker.

\subsection{Exit Choice}

A research goal was to study the initial exit choice, i.e., stairs versus elevators. The results are shown in Table 2 . It can be seen that a vast majority chose to evacuate using the elevator in all scenarios. In total, 95.5\% $(n=63)$ of the participants who evacuated chose the elevator as their escape route. From the results, it is not possible to find any statistical differences between the preferred exit choice when comparing the different scenarios with a Fischer's exact test, $p=0.767$.

As mentioned in the section on experiment setting, one of the elevators could be reached directly from the end of the corridor. This elevator was used by $9.1 \%$ $(\mathrm{n}=2)$ in scenario $1-\mathrm{FL}, 18.2 \%(\mathrm{n}=4)$ in scenario 1 and $22.7 \%(\mathrm{n}=5)$ of the participants in scenario 2. Fischer's exact test show no statistical difference between the three scenarios in terms of usage of this elevator, $p=0.451$. However, it should be noted that the scenario with the highest selection of this elevator was the scenario starting in hotel room 2, which was the room farthest away from this elevator.

As the perception of the escape routes could be an important factor for the exit choice, a question in the follow-up questionnaire was if the participants noticed more than one escape route. The results from this question are shown in Table 3. It can be seen that $22.7 \%$ of the participants $(n=15)$ noticed more than one escape route. In scenario 2, where the door to the staircase had to be passed to reach the elevator lobby, most participants noticed that there were more alternatives at hand. However, no statistically significant difference between the scenarios can be seen when analysing these results with Fischer's exact test, $p=0.258$. 
In the questionnaire, the participants were asked in a free text question why they chose the exit they had used. The most common factors given in the answers are listed below:

- because of the information in the evacuation alarm $(37.8 \%, \mathrm{n}=25)$,

- it was the way I entered the building $(22.7 \%, \mathrm{n}=15)$,

- it was the closest exit $(16.7 \%, \mathrm{n}=11)$, and

- it was the fastest way out $(16.7 \%, \mathrm{n}=11)$.

It should be noted here that the proportions of participants who answered that they choose the elevator because it was the closest exit was $13.6 \%(\mathrm{n}=3)$ in scenario $1,27.3 \%(n=6)$ in scenario $1-\mathrm{FL}$ and $9.1 \%(\mathrm{n}=2)$ in scenario 2 .

The participants were also asked what risks they perceived to be associated with their escape route. The most common answers among those who choose the elevator were:

- waiting time $(33.3 \%, \mathrm{n}=22)$,

- getting stuck in the elevator $(28.8 \%, \mathrm{n}=19)$ and,

- smoke and flames could enter the elevator $(13.6 \%, \mathrm{n}=9)$.

Also, 30.3\% $(\mathrm{n}=20)$ answered that they did not reflect on any risks associated with the elevator. Note that the participants could give multiple answers to this question.

The three participants who chose the staircase for evacuation had the following explanation for their choice of escape route:

- the voice alarm said not to use the elevators (participant in scenario 1-FL)

- it was the closest escape route (participant in scenario 2)

- wanted to use the elevator as a first choice because it seemed faster but then I went for the stairs (participant in scenario 2)

Note specifically that the one participant who choose the staircase in scenario 1FL misunderstood the message in the evacuation alarm (see the first bullet above).

\subsection{Movement Paths}

The closed fire door to the elevator lobby created some confusion among many of the participants. A total of $62.1 \%(n=36)$, passed the door and walked along the corridor to then turn back when they realised that they had walked too far. Some participants passed the door several times before finding it and reaching the elevator lobby. An example of a walking path in the scenario 1 is given in Fig. 5. The number of participants who passed the door was higher in scenario 2 where the participants had to walk longer in the corridor to reach the elevator lobby, which can be seen in Table 4. However, Fischer's exact test did not show any statistical difference between the three scenarios, $p=0.889$. It should be noted here that 10 out of the 11 participants who choose the elevator from the corridor, in all scenarios, passed the elevator lobby door during their evacuation. 
with Evacuation Elevators

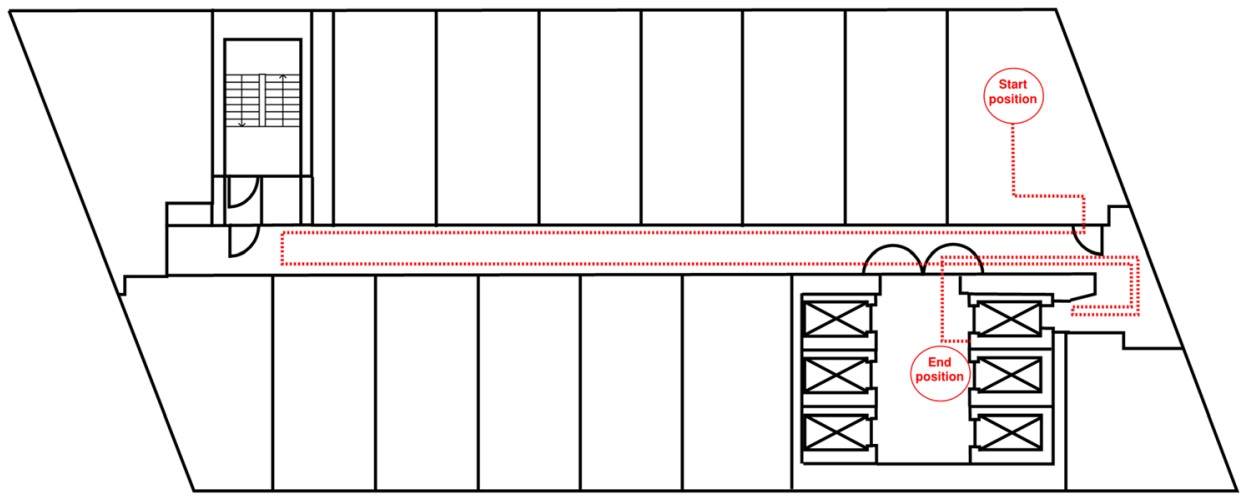

Figure 5. Schematic movement path for a participant in scenario 1 where the participant passed the elevator lobby doors several times.

\section{Table 4}

Number of Participants Who Passed the Door to the Elevator Lobby

\begin{tabular}{llll}
\hline \multirow{2}{*}{ Scenario } & \multicolumn{2}{c}{ Passed the door to the elevator lobby? } & \\
\cline { 2 - 3 } 1 & Yes, n (\%) & No, n (\%) & Total \\
\hline $1-$ FL & $11(57.9)$ & $8(42.1)$ & 19 \\
2 & $11(64.7)$ & $6(35.3)$ & 17 \\
\hline
\end{tabular}

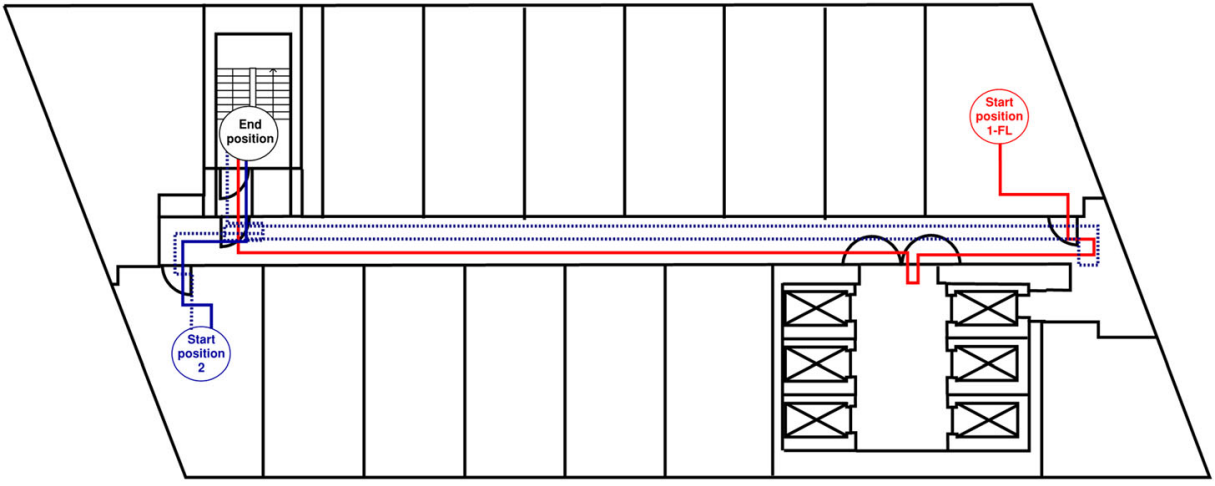

Figure 6. Schematic movement paths for the participants who chose to use the evacuation stair. The starting points for the participants in the scenarios are marked with 1 -FL and 2 in the figure. 
For the three participants who decided to evacuate through the evacuation staircase, the walking paths are visualised in Fig. 6. It can be seen that one participant in scenario 2 walked straight to the evacuation staircase. The other participant who choose the staircase walked all the way across the corridor and then turned back before choosing to use the staircase. Also, it can be seen that the participant who chose the staircase in scenario 1-FL first entered the elevator lobby, but then chose to change to the staircase before pressing the elevator call button. This participant explained to one of the researchers after the experiment that he/ she had misinterpreted the voice evacuation alarm and believed that it said explicitly NOT to use the elevators for evacuation (see also Sect. 3.2).

\subsection{Eye-Tracking Data}

Most participants fixated their gaze on the evacuation signage in some way during evacuation. For comparative and illustrative purposes, the relative number of fixations for each of the three evacuation signs in the corridor, i.e., the sign towards the elevator lobby, the fluorescent sign on the lobby door and the sign towards the staircase, is shown as a heat map in Fig. 7. The heat map has been created using Fig. 2 as a background and illustrates the differences in fixation counts on the signs between the studied scenarios. Here, it can be seen that there where relatively more fixations on the sign towards the elevator lobby in scenario 1-FL compared to scenario 1 and scenario 2. Also, the sign towards the staircase (in the end of the corridor in Fig. 7) had relatively more fixations in scenario 2 compared to scenario 1 and scenario 1-FL. This data is described more in detail in Tables 5 and 7 below.

The number of participants who fixated their gaze on the sign in scenario 1-FL can be compared to scenario 1 to explore the influence of the flashing lights. Fischer's exact test show a statistical significant difference in the number of participants who fixated their gaze on the evacuation sign, $p=0.008$. The participants in scenario 2 had a different scenario setup and are therefore not included in the

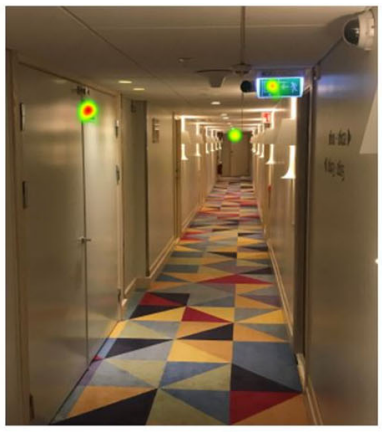

Scenario 1

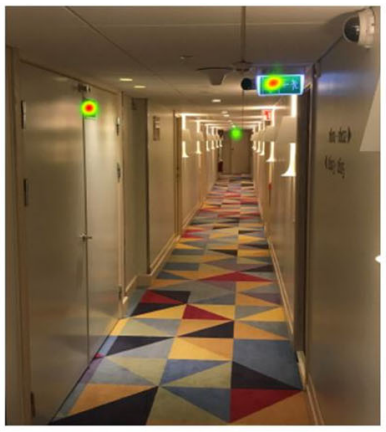

Scenario 1-FL

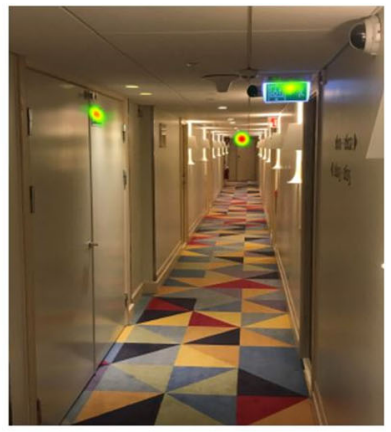

Scenario 2

\section{Figure 7. Schematic heat maps illustrating the number of fixations for each of the evacuation signs in the different scenarios. The colouring is relative with red meaning more fixations and green meaning less.}




\section{Table 5 \\ Number of Participants Who Fixated Their Gaze on the Evacuation Signs in the Corridor}

\begin{tabular}{lllr}
\hline & \multicolumn{2}{c}{ Fixated their gaze on the sign towards } & \\
\cline { 2 - 3 } Scenario & The elevator lobby, $\mathrm{n}(\%)$ & The staircase, $\mathrm{n}(\%)$ & Total \\
\hline 1 & $7(36.8)$ & $4(21.1)$ & 19 \\
$1-\mathrm{FL}$ & $14(82.4)$ & $5(29.4)$ & 17 \\
2 & $9(40.9)$ & $8(36.4)$ & 22 \\
\hline
\end{tabular}

statistical analysis. However, the proportion of participants who fixated their gaze on the sign in this scenario was similar to scenario 1 .

Also, for the participants who fixated their gaze on a sign in the corridor, it was investigated how many of these participants who also complied with the guidance given by these signs, see Table 6 . To measure the compliance, it was recorded if the participant followed the instructions on the sign during their first passage past the sign after they fixated their gaze on it. Whether the flashing lights had any effect on the compliance could be tested by comparing the compliance to the sign pointing towards the elevator lobby in scenario 1 and scenario 1-FL. Fischer's exact revealed no statistical significance, $p=0.64$. It should also be noted that all the participants who chose the staircase did so after fixating their gaze on the evacuation sign pointing towards the staircase. However, not all participants who fixated their gaze on this sign complied with this guidance.

Except for the number of participants fixating their gaze on the sign towards the elevator lobby with or without flashing lights, the times of the fixations on this sign can be studied. These are illustrated in a boxplot below (see Fig. 8). For these fixation times, scenario 1 had an average fixation time of $0.68 \mathrm{~s}$ with a SD of $0.75 \mathrm{~s}$, and a median of $0.44 \mathrm{~s}$. Scenario 1-FL had an average fixation time of $1.22 \mathrm{~s}$, a SD of $1.28 \mathrm{~s}$ and a median of $0.84 \mathrm{~s}$. Scenario 2 had an average fixation time of $0.71 \mathrm{~s}$, an SD of $0.62 \mathrm{~s}$ and a median of $0.54 \mathrm{~s}$. Again, scenario 1 and sce-

\section{Table 6}

Number of Participants Who Complied with the Signs in the Corridor

\begin{tabular}{lll}
\hline & \multicolumn{2}{c}{ Complied with the sign towards } \\
\cline { 2 - 3 } Scenario & The elevator lobby, n $(\%)$ & The staircase, $\mathrm{n}(\%)$ \\
\hline 1 & $3(42.9)$ & $0(0.0)$ \\
$1-\mathrm{FL}$ & $4(28.6)$ & $1(20.0)$ \\
2 & $3(33.3)$ & $2(25.0)$ \\
\hline
\end{tabular}




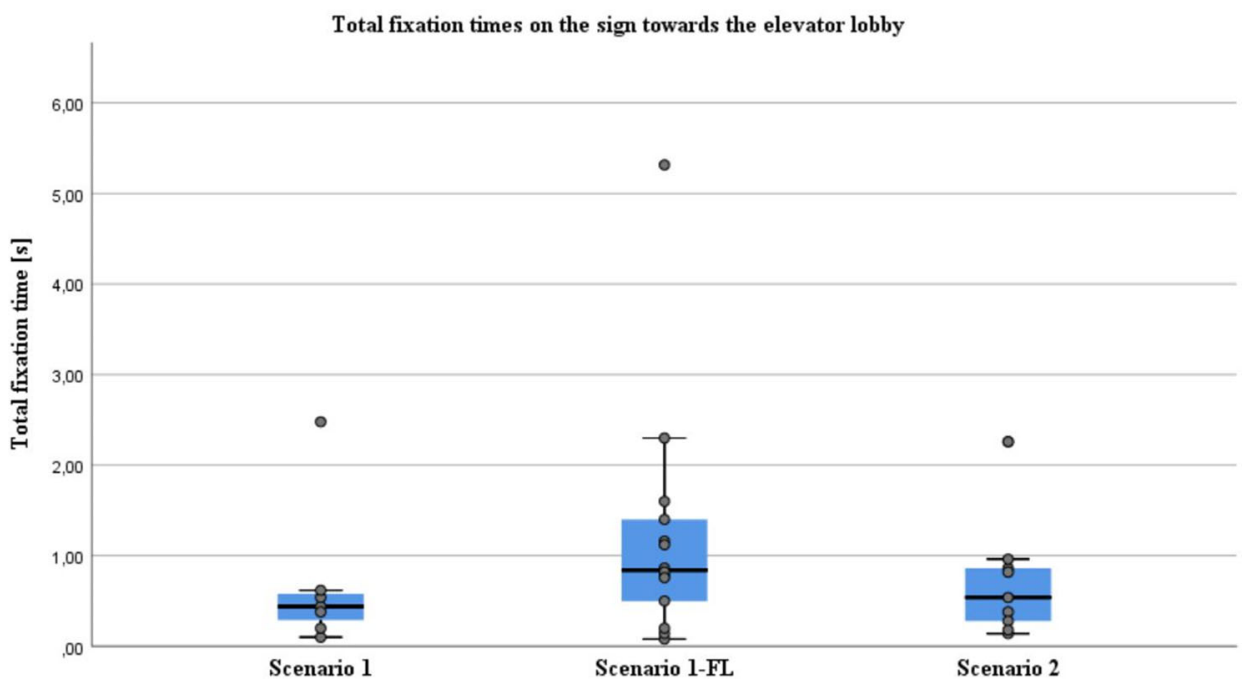

Figure 8. The fotal fixation times on the sign pointing towards the elevator lobby in each scenario. The overlaying scatter shows all data points collected in each scenario.

nario 1-FL have the same scenario setup and comparing the fixation times between these scenarios using a Mann-Whitney U test, a statistically significant difference could not be concluded, $\mathrm{U}=30.5, p=0.18$, even if a trend towards longer times for flashing lights can be seen in the boxplot (Fig. 8).

As mentioned in Sect. 2.2, there was also a fluorescent sign put on the door that led to the elevator lobby. The number of participants who fixated their gaze on this sign is given in Table 7. When comparing these results using a Chi square test, no statistical significant difference could be detected, $\chi^{2}(2, \mathrm{~N}=58)=2.211$, $p=0.331$. It should be noted that the fixations on this sign occurred, in many cases, during or just before the participants opened the door. Hence, the head position of the participant could have an important role as participants who looked straight ahead or slightly upwards would most often notice the sign as it

\section{Table 7}

Number of Participants Who Fixated Their Gaze on the Fluorescent Evacuation Sign on the Door to the Elevator Lobby

\begin{tabular}{llll}
\hline & \multicolumn{2}{l}{ Fixated their gaze on the sign on the door? } \\
\cline { 2 - 4 } Scenario & Yes, n (\%) & No, n (\%) & Total \\
\hline 1 & $13(57.9)$ & $6(42.1)$ & 19 \\
$1-\mathrm{FL}$ & $12(70.6)$ & $5(29.4)$ & 17 \\
2 & $11(50.0)$ & $11(50.0)$ & 22 \\
\hline
\end{tabular}


was a contrast to the otherwise white door. Participants who looked at the handle or slightly downwards, did not notice this sign to the same extent.

\section{Discussion}

In this study, almost all participants chose to evacuate using the elevators, regardless of the scenario studied. This is not in line with previous research on elevator evacuation, which indicate lower acceptance for the elevator as an escape route. The correlations presented in previous studies predicts the willingness for OEE usage on the 16th floor of a building to $14.5 \%$ [15], 23.5\% [14] and 44.7\% [13]. The VR study performed in a similar (but virtual) environment resulted in $59.6 \%$ of the participants using the elevators when the exit signage was not reinforced with a green flashing light and $90.0 \%$ of the participants when the exit signage was reinforced with such a light [16]. The experiment suggests that past studies have underestimated the willingness to use elevators for evacuation in high-rise hotel buildings. However, as previous studies indicate that the willingness to use the elevator is floor dependent, it should be noted that the results would likely be different if experiments were performed on another floor. This is a topic which should be further investigated in future studies.

It should be noted that the evacuation alarm contained information that the elevators could be used for evacuation, which likely affected the behaviour. However, the evacuation alarm in this study gave the same information as was given by the evacuation alarm in the VR study [16]. Also, participants in the questionnaire studies [13-15] were informed that the elevators were possible to use for evacuation. Hence, information that the elevators can be used for evacuation per se cannot explain the difference in exit choice between studies. That said, future studies should investigate how instructions in the voice alarms affects the choice of evacuation route in this sort of situation.

As the willingness to use the elevators was high in all scenarios, no difference in exit choice could be seen when comparing the results of these scenarios. Thus, a significant influence of flashing green light on exit choice could not be shown, which was the case in the VR study [16]. Neither was there any significant difference between the exit choice of participants depending on start position on the hotel floor. This suggests that the primary drive for a person in an evacuation situation may be to return the way she or he entered the building. This is coherent with the Theory of Affiliation [12], i.e., movement towards the familiar, which is a well-established theory in the area of human behaviour in fire.

The results of this study indicate that a reason for choosing the familiar route to evacuate could be that the evacuees are not aware of any other way to safety (see data in Table 3). This highlights the need for efficient way-finding systems in buildings where the evacuation strategy contradicts the normal use of the building. It should also be noted that the experiments were performed in a hotel building and that the participants did not have any previous knowledge of the building, i.e., participants were not more familiar with evacuation routes than everyday hotel guest. If participants would have been more familiar with the evacuation 
routes, e.g., as in an office or residential building, the results may have been different.

The self-closing fire door to the elevator lobby affected the evacuation as more than half of the participants passed the closed door at least once during evacuation. This was likely because the door was white, which was the same colour as the walls, and did not stand out in any other way except for the fluorescent evacuation sign on the door. These results indicate that a change along the known route, e.g., closing of a fire door, can affect the evacuation negatively. This change of the physical environment could be part of the explanation of why some participants choose the elevator from the corridor. Almost all participants who choose this elevator passed the elevator lobby door during evacuation. This indicates that such a change could force evacuees to search for alternate routes, which could possibly extend the total evacuation time in some cases.

As for pre-evacuation times, the times recorded in this experiment are relatively short compared to previous studies [37]. However, participants were awake and did not have any luggage, which likely decreases the pre-movement time compared to that of actual hotel guests. Thus, the pre-evacuation times should not be considered representative for hotel buildings in general.

Before the alarm was activated, most participants did not fixate their gaze on the evacuation signage. Similarly, the evacuation plan and the safety instructions were only studied by a few participants. Again, the limited time in the hotel room, the lack of personal belongings and the perceived reason for being in the room could probably affect the behaviour in the hotel room. However, after the alarm was activated, most participant looked at the alarm speaker as it provided the evacuation message. Also, many participants looked for information on the evacuation plan on the door before exiting the hotel room. This behaviour is coherent with previous research on human behaviour in fires [38].

The results from the eye-tracking data also show that the flashing green light had a significant impact on the noticeability of the sign. Also, the flashing light seems to increase the total time that participants fixated their gaze on the sign, even if statistical significance could not be shown. These factors should increase the understanding of the sign. However, the number of participants passing the self-closing door to the elevator lobby was not lower in the flashing lights scenario compared to the scenario without flashing lights. This indicates that, even though the green flashing light promotes the participants to notice the evacuation signage, it does not necessarily increase the compliance with the instructions of the sign.

For the eye-track data on sign noticeability, scenario 1 and scenario 1-FL were primarily compared. The reason for this was that these scenarios had the same setup, except for the flashing lights attached to the evacuation signage towards the elevator lobby. Studying the results for the sign towards the elevator lobby in scenario 2, the participants displayed longer travel paths in the corridor, meaning that they had more time to discover and look at the sign compared to the other scenarios. As for the sign towards the staircase, the proportion of participants who fixated their gaze on this sign was similar in all scenarios. This could be explained by the sign being close to the hotel room door in scenario 2, which lead 
to many participants passing under it without noticing it on their passage through the corridor.

The experiments were performed individually, i.e., social influence was not studied. It could be argued that this is an improbable evacuation scenario. However, in a hotel building, this could be expected to occur in certain cases, e.g., when occupancy is low. Also, one objective of the study was to investigate exit choice between OEEs and stairs, which is something that has not been studied in this way previously. Individual trials were deemed most suitable to be able to study this factor in isolation and without confounding factors, e.g., social influence. However, future research should investigate how social influence affects exit choice in this type of setting.

As mentioned, the method used in this study was an unannounced evacuation experiment with partly informed participants. The participants were informed they were part of a research study, but were unaware of the true purpose, i.e., the evacuation part. This method could be argued to have high external validity since participants are unaware of the evacuation and therefore can be assumed to behave in a manner similar to what could be expected in a real evacuation [17]. However, it should be noted that the participants were not regular hotel guests, which naturally affects the validity of the experiments. Also, the method does come at a price, namely the ethical dilemma of deception research. Because of this, the study went through a thorough process to ensure that the ethical aspects were considered properly. This is further described in the section on ethical approval below.

It should also be noted that the questionnaire was answered by the participants shortly after their evacuation. Thus, stress or other feelings experienced during the evacuation may have influenced their recollection of the experiment. This factor will always be present for this sort of experiment and due to the nature of the questions asked, it was deemed best to ask the participants to answer the questionnaire as soon after the experiment as possible. However, all participants were debriefed before the questionnaire and during the debriefing the researcher made sure that the participant calmed down and were ready to answer the questions.

\section{Conclusions}

The results of the study show that the willingness to use elevators for evacuation in a high-rise hotel building is higher than predicted by previous research. Furthermore, the results suggest that people who use an elevator to enter this type of building and are given the information that the elevator can be used for evacuation in the alarm message, will likely be willing to use elevators for evacuation. However, it should be noted that this study was limited to initial exit choice and that other factors that could affect exit choice were not included, e.g., elevator waiting time, social influence, etc.

The results from the eye-tracking analysis show that most building visitors do not fixate on evacuation signage before an evacuation alarm is triggered. When the alarm is activated, people fixate their gaze on signage to a greater extent than 
before the alarm, but many do still not look at evacuation signs. If the evacuation signage is reinforced with a green flashing light, the number of persons fixating on the sign increases significantly. Also, the amount of time fixating the sign reinforced with flashing lights increases as well. However, the fact that more people fixate their gaze on the signage, and do so under longer times, does not mean that they understand or conform to the information conveyed by the sign to a greater extent.

Finally, the results suggests that self-closing fire doors without vision panels can affect the evacuation and confuse evacuees. This can potentially lead to longer evacuation times in some cases.

\section{Acknowledgments}

This paper was funded by $̊$ Forsk (Grant Number 16-597) in Sweden. The authors would like to thank Richard Palmu and Scandic hotels for providing the venue (hotel) for the experiments. The authors would also like to thank Alexander Elias, Daniel Håkansson, Markus Nilsson and Martin Forssberg for their support during the experiments and Carl-Johan Herbst for reviewing the Swedish research report. The authors would also like to thank Dr. Håkan Frantzich, Dr. Enrico Ronchi and Dr. Robert Jansson-McNamee for valuable feedback on this manuscript.

\section{Funding}

Open access funding provided by Lund University.

\section{Compliance with ethical standards}

Ethical approval This study was performed with human participants. Thus, much of the experimental planning was devoted to participant safety and ethical considerations. Before the experiments were conducted, an ethics application was sent to the Swedish Ethical Review Authority, and ethical approval was granted. All participants gave consent prior to the experiment, but did not the true purpose of the experiment at this point. After the experiment, the participants were debriefed, and all gave a fully informed consent. The consent given before the experiment did not include the information that evacuation was part of the study. For this reason, participants had to give a second informed consent after the study to approve the use of the data collected. Also, participants were reimbursed with two movie tickets (approximate value of 250 SEK) after the experiment, regardless of the experiment being terminated or not. The videos and questionnaires were anonymised so that participants could not be linked to the results after the experiment. 


\section{Open Access}

This article is licensed under a Creative Commons Attribution 4.0 International License, which permits use, sharing, adaptation, distribution and reproduction in any medium or format, as long as you give appropriate credit to the original author(s) and the source, provide a link to the Creative Commons licence, and indicate if changes were made. The images or other third party material in this article are included in the article's Creative Commons licence, unless indicated otherwise in a credit line to the material. If material is not included in the article's Creative Commons licence and your intended use is not permitted by statutory regulation or exceeds the permitted use, you will need to obtain permission directly from the copyright holder. To view a copy of this licence, visit http://creat ivecommons.org/licenses/by/4.0/.

\section{References}

1. McNamee $\mathbf{M}$ et al (2019) IAFSS agenda 2030 for a fire safe world. Fire Saf J 110:102889. https://doi.org/10.1016/j.firesaf.2019.102889

2. World's Tallest Buildings_-SkyscraperPage.com. http://skyscraperpage.com/diagrams/?s earchID $=200$. Accessed 17 Jun 2019

3. World Skyscraper Construction-SkyscraperPage.com. https://skyscraperpage.com/diag rams/?searchID $=202$. Accessed 17 Oct 2019

4. United Nations, Department of Economic and Social Affairs, and Population Division (2019) World population prospects highlights, 2019 revision highlights, 2019 revision

5. Boyce KE (2017) Safe evacuation for all - fact or fantasy? Past experiences, current understanding and future challenges. Fire Saf J 91:28-40. https://doi.org/10.1016/j.firesaf.2017.05.004

6. Ronchi E, Nilsson D (2014) Modelling total evacuation strategies for high-rise buildings. Build Simul 7(1):73-87

7. Kuligowski ED (2003) Elevators for occupant evacuation and fire department acces. In: Presented at the CTBUH/CIB Kuala Lumpur 2003 conference, Kuala Lumpur

8. Siikonen M-L, Hakonen H (2002) Efficient evacuation methods in tall buildings. In: Presented at the ELEVCON, the international congress on vertical transportation technologies, Milan

9. Kinsey MJ, Galea ER, Lawrence PJ (2009) Investigating the use of elevators for highrise building evacuation through computer simulation. In: Presented at the 4th international symposium on human behaviour in fires, Cambridge

10. Reneke PA, Peacock RD, Hoskins BL (2013) Combined stairwell and elevator use during building evacuation. National Institute of Standards and Technology, NIST TN 1793. https://doi.org/10.6028/nist.tn.1793

11. Ronchi E, Nilsson D (2013) Fire evacuation in high-rise buildings: a review of human behaviour and modelling research. Fire Sci Rev. https://doi.org/10.1186/2193-0414-2-7

12. Sime JD (1985) Movement toward the familiar-person and place affiliation in a fire entrapment setting. Environ Behav 17(6):697-724

13. Kinsey MJ, Galea ER, Lawrence PJ (2012) Human factors associated with the selection of lifts/elevators or stairs in emergency and normal usage conditions. Fire Technol 48(1):3-26. https://doi.org/10.1007/s10694-010-0176-7 
14. Heyes E, Spearpoint M (2009) Lifts for evacuation-human behaviour considerations. Fire Mater 2009:73-84

15. Jönsson A, Andersson J, Nilsson D (2012) A risk perception analysis of elevator evacuation in high-rise buildings. Cambridge, pp 398-409

16. Andrée K, Nilsson D, Eriksson J (2016) Evacuation experiments in a virtual reailty high-rise building: exit choice and waiting time for evacuation elevators. Fire Mater 2016(40):554-567

17. Nilsson D (2009) Exit choice in fire emergencies - influencing choice of exit with flashing lights. Lunds Universitet, Lund, Doktorsavhandling 1040

18. Ronchi E et al (2016) A virtual reality experiment on flashing lights at emergency exit portals for road tunnel evacuation. Fire Technol 52(3):623-647. https://doi.org/10.1007/ s10694-015-0462-5

19. Butler KM, Furman SM, Kuligowski ED, Peacock RD (2016) Perspectives of occupants with mobility impairments on fire evacuation and elevators. National Institute of Standards and Technology, NIST TN 1923. https://doi.org/10.6028/nist.tn.1923

20. Duchowski AT (2017) Eye tracking techniques. In: Duchowski AT (ed) Eye tracking methodology: theory and practice Springer, Cham, pp 49-57

21. Rucci M, Poletti M (2015) Control and functions of fixational eye movements. Annu Rev Vis Sci 1(1):499-518. https://doi.org/10.1146/annurev-vision-082114-035742

22. Kowler E (2011) Eye movements: the past 25 years. Vis Res 51(13):1457-1483. https:// doi.org/10.1016/j.visres.2010.12.014

23. Carrasco M (2011) Visual attention: the past 25 years. Vis. Res 51(13):1484-1525. https://doi.org/10.1016/j.visres.2011.04.012

24. Sadasivan S, Greenstein JS, Gramopadhye AK, Duchowski AT (2005) Use of eye movements as feedforward training for a synthetic aircraft inspection task. In: Proceedings of the SIGCHI conference on Human factors in computing systems-CHI'05, Portland, Oregon, USA, p 141. https://doi.org/10.1145/1054972.1054993

25. Balk SA, Moore KS, Steele JE, Spearman WJ, Duchowski AT (2006) Mobile phone use in a driving simulation task: differences in eye movements. J Vis 6(6):872-872. https://doi.org/10.1167/6.6.872

26. Granka LA, Joachims T, Gay G (2004) Eye-tracking analysis of user behavior in WWW search. In: Proceedings of the 27th annual international ACM SIGIR conference on research and development in information retrieval, New York, NY, USA, pp 478-479. https://doi.org/10.1145/1008992.1009079

27. Till RC, Babcock JS (2011) Proof of concept: use of eye-tracking to record how people use exit signage. In: Pedestrian and evacuation dynamics, Boston, MA, pp 209-219. htt ps://doi.org/10.1007/978-1-4419-9725-8_19

28. Li Y, Zhang P, Zhang H (2017) Study on the location of building evacuation indicators based on eye tracking. In: Proceedings of the 3rd ACM SIGSPATIAL workshop on emergency management using, Redondo Beach, CA, USA, pp 1-5. https://doi.org/10.11 $45 / 3152465.3152467$

29. Ding N, Chen T, Liu Y (2018) Evacuation guidance design: an experimental study based on eye tracking devices. In: Presented at the 9th international conference on pedestrian and evacuation dynamics (PED2018), Lund

30. How do Tobii Eye Trackers work? - Learn more with Tobii Pro (2015) https://www.tob iipro.com/learn-and-support/learn/eye-tracking-essentials/how-do-tobii-eye-trackers-wor k/. Accessed 10 Oct 2019

31. Tobii Pro Glasses 2 wearable eye tracker (2015) https://www.tobiipro.com/product-list ing/tobii-pro-glasses-2/. Accessed 10 Oct 2019 
with Evacuation Elevators

32. Tobii Pro Lab - Software for eye tracking and biometrics (2015) https://www.tobiipro.c om/product-listing/tobii-pro-lab/. Accessed 10 Oct 2019

33. Duchowski AT (2017) Taxonomy and models of eye movements. In: Duchowski AT (ed) Eye tracking methodology: theory and practice Springer, Cham, pp 39-46

34. Tobii Pro Lab (2018) When do I use the I-VT attention filter? https://connect.tobiipro.c $\mathrm{om} / \mathrm{s} /$ article/When-do-I-use-the-I-VT-Attention-filter?language $=$ en_US. Accessed 08 Jan 2020

35. Olsen A (2012) The Tobii I-VT fixation filter, p 21

36. IBM SPSS Software (2018). https://www.ibm.com/se-sv/analytics/spss-statistics-software . Accessed 16 May 2019

37. Lovreglio R, Kuligowski E, Gwynne S, Boyce K (2019) A pre-evacuation database for use in egress simulations. Fire Saf J 105:107-128. https://doi.org/10.1016/j.firesaf.2018.12.009

38. Canter D, Breaux J, Sime J (1980) Domestic, multiple occupancy, and hospital fires. In: Fires and human behaviour. Wiley, New York, pp 117-136

Publisher's Note Springer Nature remains neutral with regard to jurisdictional claims in published maps and institutional affiliations. 